\title{
Calcium Pyrophosphate Dihydrate Crystal Deposition Disease of the Sternoclavicular Joint
}

\author{
Andreas Borowski ${ }^{1}$ Sebastian Heikaus ${ }^{2}$ Muhammed Kurt ${ }^{1}$ \\ ${ }^{1}$ Department of Cardiothoracic Surgery, University of Duesseldorf, \\ Duesseldorf, Germany \\ 2 Institute of Pathology, University of Duesseldorf, Duesseldorf, \\ Germany \\ Address for correspondence Andreas Borowski, MD, Department of \\ Cardiothoracic Surgery, University of Duesseldorf, Moorenstrasse 5 , \\ Duesseldorf 40255, Germany \\ (e-mail: Borowski@med.uni-duesseldorf.de).
}

Thorac Cardiovasc Surg Rep 2015;4:46-48.

\begin{abstract}
Deposition of calcium pyrophosphate dihydrate (CPPD) crystals in the articular structures affects predominantly temporomandibular, knee, hip, spine, and wrist joints, and is a rare condition, often mimicking malignancy. Sternoclavicular joint is extremely

Keywords

- thoracic surgery

- sternoclavicular joint

- osteoarthritis

- resection arthroplasty rarely involved. We present a patient with swelling of the right upper extremity, in whom on computed tomography a mass posterior to the sternoclavicular joint causing compression of the brachiocephalic vein was detected. A modified resection arthroplasty was performed, and the histopathological findings revealed massive deposits of CPPD in the articular cartilage. To our knowledge, there is only one similar case published in the literature.
\end{abstract}

\section{Introduction}

Calcium pyrophosphate dihydrate (CPPD) disease affects para-articular soft tissues or articular components, such as cartilage, and causes painful arthritis often designated as tophaceous pseudogout. ${ }^{1}$ It commonly occurs in the temporomandibular joints, hips, and knees. Patients affected with this condition usually present with pain and swelling in the joints. Radiological finding manifests as punctate or linear radiodensities, or irregularly contoured mass and can easily be misinterpreted as a malignant lesion (i.e., chondrosarcoma). The etiology of this disease is not clear. Studies suggest that some noxious events can trigger the hypertrophy and degeneration of chondrocytes with subsequent calcium accumulation in large amounts, which together with an inflammatory reaction result in a pseudotumor mass formation. The increased incidence was observed in humans with hyperparathyroidism, hemochromatosis, hemosiderosis, and hypomagnesemia. ${ }^{2}$ We discuss this rare disease with an unusual target joint in regard to the clinical manifestation, differential diagnosis, and possible surgical therapy.

received

June 8, 2014

accepted after revision

July 3, 2014

published online

October 31, 2014

\section{Case Report}

In May 2011, a 76-year-old man with a history of insulindependent diabetes mellitus, hypertension, hypercholesterinemia, prostate cancer, and osteoarthritis of both knees, requiring total knee replacements, presented to his general practitioner with progressive swelling of the right upper extremity and prominent venous network of the neck. At phlebography, occlusion of the right brachiocephalic vein was diagnosed. Presuming that a bicycle accident in the early patient's history could be the cause, no further diagnostic procedures were initiated and Coumadin (Meda Pharma GmbH \& Co. KG, Bad Homburg, Germany) was administered as a therapy. Three months later, computed tomography (CT) and magnetic resonance (MR) imaging performed at our center revealed a mass posterior to the right sternoclavicular joint with subtotal compression of the right brachiocephalic vein (-Figs. 1 and 2). Neither local signs of tumor (protuberance) could be seen, nor pain sensation was reported by the patient, nor could be provoked by palpation. Laboratory investigations revealed no pathology (especially regarding

\footnotetext{
(c) 2015 Georg Thieme Verlag KG Stuttgart · New York
}

\section{License terms}

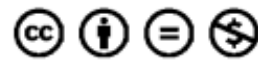




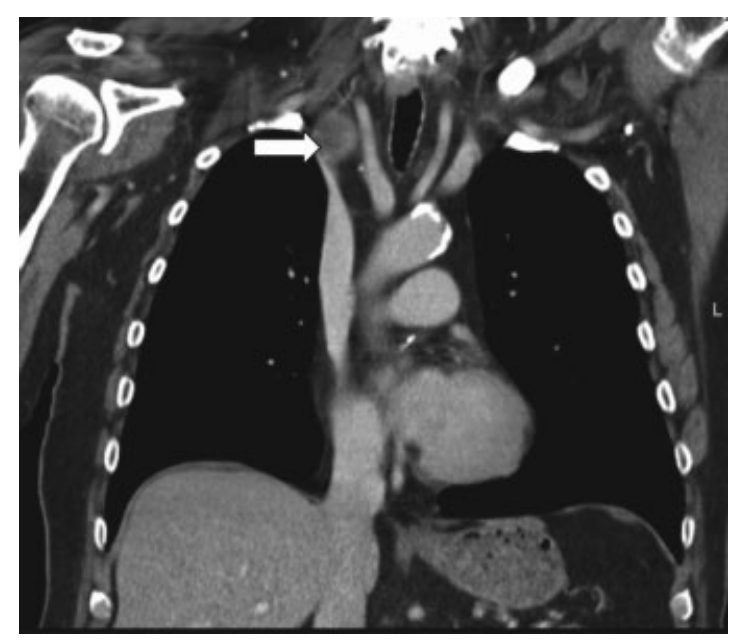

Fig. 1 Contrast-enhanced computed tomography (CT) scan shows a large mass posterior to the right sternoclavicular joint (arrow) causing compression of the brachiocephalic vein.

thyroid, parathyroid and kidney functions, alkaline phosphatase, calcium, magnesium, phosphate, transferrin saturation and ferritin, and rheumatoid factors) with the exception of a slightly increased inflammatory marker (CRP $2.3 \mathrm{mg} / \mathrm{dL}$ ). A specimen of the CT-guided biopsy of the sternoclavicular joint was negative for infection and malignancy. A modified Rockwood resection arthroplasty ${ }^{3}$ with upper longitudinal Jshaped sternotomy to facilitate resection (see schematic drawing in - Fig. 3) was performed, and the histopathological specimens of the resected joint revealed CPPD crystals in the cartilage with associated high-degree synovialitis (-Fig. 4). The patient had an uneventful postoperative course with complete recovery from arm swelling and was discharged from the hospital on the 10th postoperative day.

\section{Discussion}

Kohn and coworkers first described a condition with deposition of CPPD crystals in the synovial fluid in patients presenting with gout-like symptoms and designated it as a pseudogout. ${ }^{4}$ According to the report of Ishida, ${ }^{1}$ a total number of 29 cases with tophaceous pseudogout have been published until 1995. Kato, ${ }^{5}$ summarizing further singular

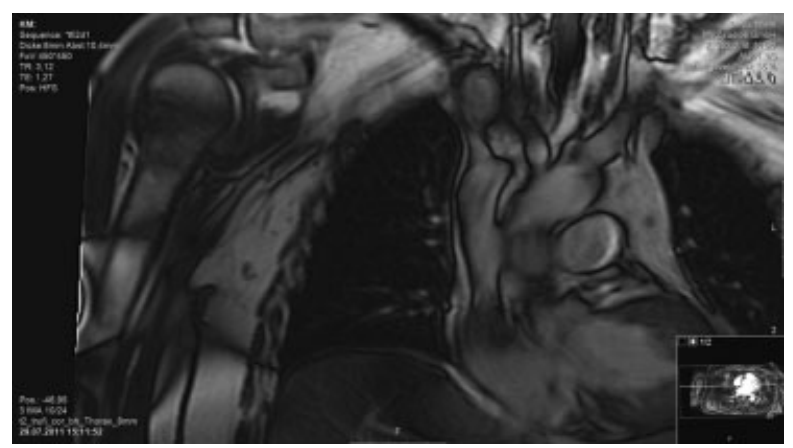

Fig. 2 Magnetic resonance (MR)-image indicates a low-signal mass extending posteriorly to the right sternoclavicular joint.

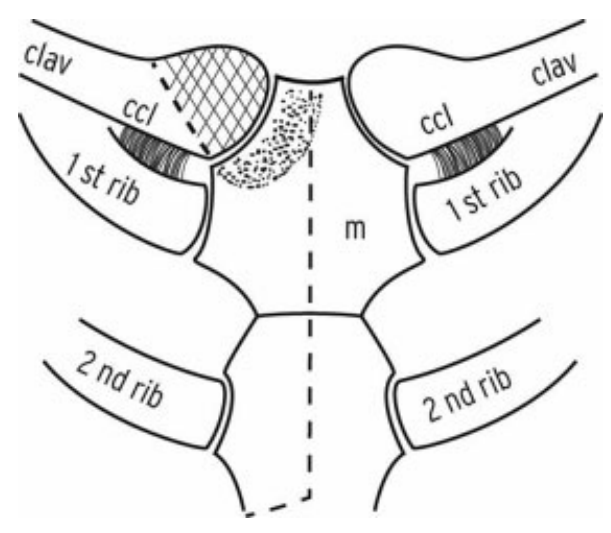

Fig. 3 Schematic drawing of surgical technique (Media Centre, Enterprise Communications, University of Düsseldorf) adapted from Rockwood $^{1}$ and modified by J-shaped sternotomy. Cross-hatched and doted areas indicate resection zone. clav, clavicle; ccl, costo-clavicular ligament; m, manubrium sterni.

cases published between 1995 and 2008, concluded that the usual site of involvement is predominantly the temporomandibular joint, but also knee, hip, and spine joints can be affected. To our knowledge, only one case with CPPD-associated arthritis of the sternoclavicular joint has been published previously. ${ }^{6}$ Interestingly, the authors reported a similar case in regard to the symptoms and history, as their patient did not suffer from arthralgia, did not present with local joint swelling, and the periarticular mass expanded posteriorly to the joint causing deviation of the brachiocephalic vein. He also had the same background morbidity with diabetes mellitus and osteoarthritis of both knees requiring joint replacements.

The exact mechanism causing CPPD remains unknown. The clinical spectrum ranges from asymptomatic (lanthanic) CPPD throughout acute pseudogout, osteoarthritis, pseudorheumatoid, and pseudoneuropathic arthritis. Generally, there is no specific therapy for CPPD and management depends on clinical manifestation. Treatment for asymptomatic CPPD is not advised; however, underlying pathologic condition supporting progressive development of the disease should be searched for and treated to prevent further damage. In case of pseudogout attacks, the anti-inflammatory agents

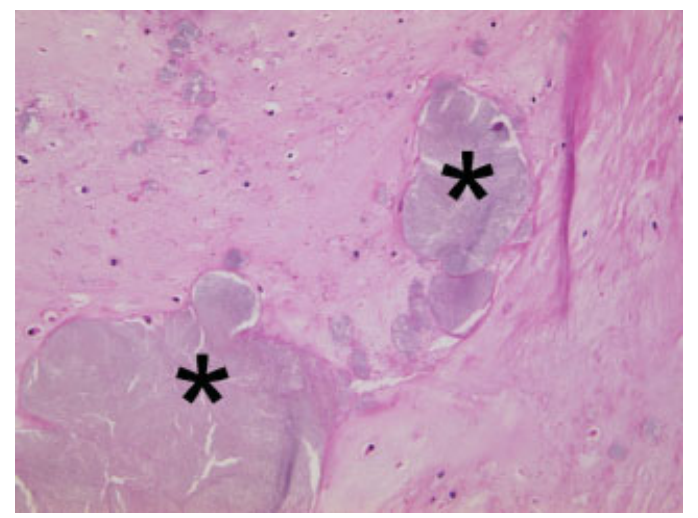

Fig. 4 Histological specimen revealed focal calcified deposits in the chondroid tissue (asterisks). 
such as corticosteroids, nonsteroidal anti-inflammatory drugs (NSAIDs) (e.g., COX-2-inhibitors), or colchicine have proved to be beneficial. Due to the unpredictable dynamics of the disease, surgical therapy should be considered only in patients who are severely disabled by this condition. In the detection and follow-up strategy, examination of synovial fluid for CPPD crystals and radiography is a diagnostic standard. When non-weight-bearing joints are affected by CPPD, radiography of knee and spine joints (as a common manifestation site) is recommended.

In summary, the arthritis of the sternoclavicular joint in the course of CPPD disease is an extremely rare condition which might proceed without arthralgia, and the first symptoms of which can be the compression of adjacent structures by the bulky joint mimicking tumor, thus requiring surgery. Multifocal osteoarthritis should alert physicians to possible CPPD.

\section{References}

1 Ishida T, Dorfman HD, Bullough PG. Tophaceous pseudogout (tumoral calcium pyrophosphate dihydrate crystal deposition disease). Hum Pathol 1995;26(6):587-593

2 Ea HK, Lioté F. Advances in understanding calcium-containing crystal disease. Curr Opin Rheumatol 2009;21(2):150-157

3 Rockwood CA Jr, Groh GI, Wirth MA, Grassi FA. Resection arthroplasty of the sternoclavicular joint. J Bone Joint Surg Am 1997; 79(3):387-393

4 Kohn NN, Hughes RE, McCarty DJ Jr, Faires JS. The significance of calcium phosphate crystals in the synovial fluid of arthritic patients: the "pseudogout syndrome". II. Identification of crystals. Ann Intern Med 1962;56:738-745

5 Kato H, Nishimoto K, Yoshikawa T, Kusuzaki K, Sudo A. Tophaceous pseudogout in the knee joint mimicking a soft-tissue tumour: a case report. J Orthop Surg (Hong Kong) 2010;18(1):118-121

6 Richman KM, Boutin RD, Vaughan LM, Haghighi P, Resnick D. Tophaceous pseudogout of the sternoclavicular joint. AJR Am J Roentgenol 1999;172(6):1587-1589 